\title{
What Does the COVID-19 Pandemic Mean for Rheumatology Patients?
}

\section{Janet E. Pope, MD, MPH, FRCPC}

\author{
Address \\ ${ }^{1}$ Schulich School of Medicine \& Dentistry, London, ON, Canada \\ ${ }^{2}$ Western University, London, ON, Canada \\ Department of Medicine, Division of Rheumatology, St Joseph's Health Care, 268 \\ Grosvenor St., London, 0N, N6A 4V2, Canada \\ Email: janet.pope@sjhc.london.on.ca
}

Published online: 30 April 2020

(C) Springer Nature Switzerland AG 2020

Keywords COVID-19 $\cdot$ Rheumatology $\cdot$ Rheumatologists $\cdot$ Coronavirus $\cdot$ Pandemic $\cdot$ Drug shortages

\begin{abstract}
Purpose of review The COVID-19 outbreak has resulted in uncertainty for patients with autoimmune rheumatic diseases for several reasons. They are concerned about their risk of developing COVID-19 as many are immune suppressed from their disease and/or treatment, whether they should stop their advanced therapies, if they will have a worse outcome if/when infected due to their underlying medication condition(s) and if they will have drug availability, especially with press (without much data) coverage suggesting hydroxychloroquine may be used in COVID-19 infection causing diversion of medication supply. This article discusses how the pandemic affects people with systemic autoimmune rheumatic diseases.

Recent findings Preliminarily, articles seem to suggest that patients with rheumatic diseases may not have more infections from SARS-CoV-2 and similar outcomes to age and gender matched patients, but fear of rheumatic medications increasing their risk, drug shortages, and work exposure all are concerns for patients.

Recent findings The long term effects of the pandemic in patients with rheumatic diseases will not be known until much later and likely include stressors flaring disease (fear, illness, job loss, social isolation), post-traumatic stress, flaring due to stopping medications, less physician visits with subsequent under-treatment, and increases in chronic concomitant fatigue, pain, fibromyalgia.
\end{abstract}

\section{Introduction}

The COVID-19 pandemic may be considered 'the cough that changed the world.' On March 11, 2020, the World Health Organization declared the COVID-19 virus a pandemic [1] which is extremely fast as the first case was reported to the WHO China office on December 31, 2019 [2]. COVID-19 is a coronavirus SARS-CoV-2 which started in Wuhan, China and it is short for coronavirus disease first found in 2019. The virus jumped species to 
humans originating in the pangolins or horseshoe bats [3]. The pangolins are widely smuggled and sold in wet markets illegally in China. The signs and symptoms of COVID-19 vary from no symptoms (carrier), to a mild respiratory tract infection to fever, cough, and bilateral pneumonitis. The latter can result in death with a varying mortality rate [4]. There are six other strains of coronavirus including four which cause only mild colds and severe acute respiratory syndrome (SARS CoV) which had an outbreak in 2003 with approximately a $10 \%$ mortality, and Middle East respiratory syndrome (MERS CoV) which is still endemic [5]. The risk factors are age, comorbidities including chronic obstructive lung disease (COPD), diabetes mellitus, and coronary artery disease. Immune-suppressed patients are also thought to be at a higher risk for worse outcomes. It is uncertain if patients with autoimmune rheumatic diseases are at a higher risk to contract the virus, and if they are infected whether they have worse outcomes. There are also different degrees of immune suppression. We can consider the host factors and treatment. Some patients will have more infections due to their rheumatic disease (such as rheumatoid arthritis, systemic lupus erythematosus, systemic sclerosis, other connective tissue diseases, and vasculitis). Additionally, corticosteroids (especially high dose) increase the risk of infection. It is less certain how much of an increased risk immune modulators such as methotrexate, azathioprine, biologics, and Janus kinase inhibitors have on the outcomes of COVID-19 but to date this is not a major risk $[6,7]$.

\section{Rheumatology concerns}

Rheumatology patients with systemic autoimmune diseases may have several concerns. They are uncertain if they should be working if they are employed in health care or other frontline jobs due to the risk of getting ill. They do not know if they should continue with their immune suppressive medications and they are concerned about drug shortages of hydroxychloroquine. The advice that is given to patients is tailored to the individual. The Canadian Rheumatology Association has a statement that includes patients should not stop their rheumatic medications unless if ill with a high fever or serious infection. They should not suddenly stop their corticosteroids if on them chronically. They should not seek medical attention for suspected COVID-19 infection unless if they meet the case definition for testing (which is region specific but may include fever and dyspnea and cough). If they go to the emergency room, they should say they are receiving medications that may increase immune suppression. Like all patients, they should cough into their arm or a tissue, wash hands frequently (for $20 \mathrm{~s}$ each time) especially after returning home if they have been out of their home, avoid touching their face (especially nose, mouth, and eyes), avoid crowds, avoid unnecessary travel, quarantine if told to do so, and to get the flu shot if not already received as influenza is still present in many jurisdictions [8].

Their infection risk is related also to the virulence of the strain (and there have been two strains of COVID-19 identified thus far) and the dose of the exposure which is why health care workers have a higher risk of poor outcomes with a high mortality already reported in Italy [9].

Other medications such as NSAIDs do not alter the course of COVID-19 despite an earlier report that ibuprofen use caused worse outcomes [10]. The conclusions were likely premature (and there could be confounding where people with worse fever or myalgias from COVID-19 would be more likely to take an NSAID than those with milder symptoms and the former would be expected to have a worse outcome. 
Antimalarial shortages (particularly hydroxychloroquine as chloroquine was already on back order in many jurisdictions) occurred after a small publication may have shown that the viral PCR decreased more in patients treated with hydroxychloroquine than controls [11]. This report contained 20 patients who were treated with hydroxychloroquine but 6 were not included of whom 1 died and 3 went to the intensive care unit and one did not tolerate the treatment. The controls were from a different hospital; so, it is difficult to interpret the data meaningfully in my opinion. This resulted in a shortage of medication. For instance, in Canada monthly, there are approximately 47,000 doses of hydroxychloroquine sold monthly and the level has been constant for 5 years, and in March, 2020 after the results of the small study were announced, the sales more than doubled in a couple of weeks causing a shortage for patients and high anxiety in many of them [12]. This is being resolved in many countries with more suppliers producing hydroxychloroquine. This has led to statements from Rheumatology organizations to ensure a constant supply to patients with rheumatic diseases [13] and a need for evidence-based treatment [14]. Despite a lack of strong data supporting antimalarial use, the WHO has facilitated a multi-arm trial (SOLIDARITY) [15] where standard of care will be compared to several other treatment arms including those randomized to hydroxy/chloroquine, or HIV antivirals (ritonavir + iopinavir) or ritonavir + iopinavir + interferon beta, or an antiviral used for Ebola (remdesivir). Not all countries will have all comparators [16].

After the pandemic is over, the world economy will be harmed and patients will have suffered from stress, income, or job loss and possibly lack of access to their care for their chronic diseases and the uncertainties of medication supply. Many people will die due to the pandemic and others may have worse outcomes from their chronic disease as they may not have access to their physicians due to quarantines and diversion of hospital resources to treat the seriously ill infected from COVID-19. Hopefully, lessons will quickly be learned about stopping the spread, communicating clear messages to our patients and providing high-quality health care to those who need it.

\section{Funding Information}

There was no financial support for this project.

\section{Compliance with Ethics Guidelines}

\section{Conflict of Interest}

The author declares that she has no conflict of interest.

\section{Human and Animal Rights and Informed Consent}

This article does not contain any studies with human or animal subjects performed by any of the authors.

\section{Ethical Approval}

There was no institutional ethics approval as this is a commentary. 


\section{References and Recommended Reading}

1. https://time.com/5791661/who-coronaviruspandemic-declaration/ referenced on April 6, 2020.

2. https://www.who.int/emergencies/diseases/novelcoronavirus-2019/events-as-they-happen referenced on April 6, 2020.

3. https://www.sciencealert.com/coronavirusdiscovery-in-pangolins-shows-why-wildlifemarkets-need-better-regulations referenced on April 6, 2020.

4. https://www.weforum.org/agenda/2020/04/we-couldbe-vastly-overestimating-the-death-rate-for-covid-19heres-why/ referenced on April 6, 2020.

5. Consensus document on the epidemiology of severe acute respiratory syndrome (SARS) https://www.who. int/csr/sars/en/WHOconsensus.pdf referenced on April 6, 2020.

6. Zhou F, Yu T, Du R, Fan G, Liu Y, Liu Z, et al. Clinical course and risk factors for mortality of adult inpatients with COVID-19 in Wuhan, China: a retrospective cohort study. Lancet, 2020;395(10229):1054-62. https://doi.org/10.1016/S0140-6736(20)30566-3.

7. Monti S, Balduzzi S, Delvino P, Bellis E, Quadrelli VS, Montecucco C. Clinical course of COVID-19 in a series of patients with chronic arthritis treated with immunosuppressive targeted therapies. Ann Rheum Dis. 2020;79(5):667-8. https://doi.org/10.1136/ annrheumdis-2020-217424.

8. https://rheum.ca/cra-updated-statement-on-covid-19march-17-2020/ referenced on April 6, 2020.

9. https://www.medscape.com/viewarticle/927753 referenced on April 6, 2020.
10. https://www.pharmacists.ca/cpha-ca/assets/File/cphaon-the-issues/Use-of-NSAIDs-in-patients-with-

COVID-19-FINAL-EN.pdf referenced on April 6, 2020.

11. Raoult D. New insights on the antiviral effects of chloroquine against coronavirus: what to expect for

COVID-19? Int J Antimicrob Agents. 2020;11:105938.

12. https://www.drugshortagescanada.ca/drugsearch? term $=$ Plaquenil\&ingredient $=\& a t c \_$code $=$\&atc descriptor $=\& \_$token $=$ IC7secHlJyidQn5XISRrpqjSE5wRYEZpspfjQ8CZ-C0 referenced on April 6, 2020.

13. https://rheum.ca/wp-content/uploads/2020/04/CRAStatement-and-FAQ-on-HCQ-supply-and-COVID-19EN.pdf) referenced on April 6, 2020.

14. https://www.cma.ca/cma-update-coronavirus?page $=1$ referenced on April 6, 2020.

15. https://www.sciencemag.org/news/2020/03/wholaunches-global-megatrial-four-most-promisingcoronavirus-treatments referenced on April 6, 2020.

16. https://clinicaltrials.gov/ct2/show/NCT04330690 referenced on April 6, 2020.

\section{Publisher's Note}

Springer Nature remains neutral with regard to jurisdictional claims in published maps and institutional affiliations. 